\title{
Reducing psychological distress and obesity in Australian farmers by promoting physical activity
}

\author{
Susan Brumby ${ }^{1,2^{*}+}$, Ananda Chandrasekara ${ }^{1,2+}$, Scott McCoombe ${ }^{1,2}$, Susan Torres ${ }^{3}$, Peter Kremer ${ }^{4}$ and \\ Paul Lewandowski
}

\begin{abstract}
Background: Studies have confirmed that the rate of mental illness is no higher in rural Australians than that of urban Australians. However, the rate of poor mental health outcomes, and in particular suicide, is significantly raised in rural populations. This is thought to be due to lack of early diagnosis, health service access, the distance-decay effect, poor physical health determinants and access to firearms. Research conducted by the National Centre for Farmer Health between 2004 and 2009 reveals that there is a correlation between obesity and psychological distress among the farming community where suicide rates are recognised as high. Chronic stress overstimulates the regulation of the hypothalamic-pituitary-adrenal (HPA) axis that is associated with abdominal obesity. Increasing physical activity may block negative thoughts, increase social contact, positively influence brain chemistry and improve both physical and mental health. This paper describes the design of the Farming Fit study that aims to identify the effect of physical activity on psychological distress, obesity and health behaviours such as diet patterns and smoking in farm men and women.

Methods/Design: For this quasi-experimental (convenience sample) control-intervention study, overweight (Body Mass Index $\geq 25 \mathrm{~kg} / \mathrm{m}^{2}$ ) farm men and women will be recruited from Sustainable Farm Families ${ }^{\mathrm{TM}}$ (SFF) programs held across Victoria, Australia. Baseline demographic data, health data, depression anxiety stress scale (DASS) scores, dietary information, physical activity data, anthropometric data, blood pressure and biochemical analysis of plasma and salivary cortisol levels will be collected. The intervention group will receive an exercise program and regular phone coaching in order to increase their physical activity. Analysis will evaluate the impact of the intervention by longitudinal data (baseline and post intervention) comparison of intervention and control groups.

Discussion: This study is designed to examine the effect of physical activity on psychological health and other comorbidities such as obesity, impaired glucose tolerance, hypertension and dyslipidaemia within a high-risk cohort. The outcomes of this research will be relevant to further research and service delivery programs, in particular those tailored to rural communities.
\end{abstract}

Trial registrationACTRN12610000827033

\section{Background}

Mental health disorders, particularly anxiety and depression, are the leading cause of health related disability in Australia [1,2]. These disorders make a large contribution to the global burden of disease, with depressive disorders being the leading cause in middle- and high-income regions such as European Union, North America, Japan and Australia [3]. By 2020, depression is projected to

\footnotetext{
* Correspondence: susan.brumby@deakin.edu.au

+ Contributed equally

'National Centre for Farmer Health, Western District Health Service, Hamilton Vic 3300, Australia

Full list of author information is available at the end of the article
}

reach second place in the global burden of disease rankings $[4,5]$. Rural Australians face a high mental health burden [6] due to social isolation [7], socio-economic constraints $[8,9]$, poor diet, increased alcohol intake, suboptimal sleep, lack of exercise, high rates of obesity [10] and diabetes [11]. As a sub-population of rural Australia it has become evident that farmers experience inferior physical and mental health than their rural counterparts $[6,12,13]$. This is also due to the distance decay effect where the further people are from a service, the longer they wait to access that service [14]. The differences between rural and farmer mental health is highlighted by the increased incidence of suicide in farming communities
C Biomed Central

() 2011 Brumby et al; licensee BioMed Central Ltd. This is an Open Access article distributed under the terms of the Creative Commons Attribution License (http://creativecommons.org/licenses/by/2.0), which permits unrestricted use, distribution, and reproduction in any medium, provided the original work is properly cited. 
worldwide [15-17]. A study of South Australian farmers revealed that the suicide rate on farms is $67 \%$ higher than in rural populations which, in turn, is higher than in urban South Australia [12]. Ongoing research with the Sustainable Farm Families ${ }^{\mathrm{TM}}$ (SFF) program reveals that poor health indicators are pronounced within the farming community [13,17-19]. Furthermore, our previous study [20] has revealed that, farmers had a higher rate of obesity/ overweight (64.3\%) when compared to national averages. Additionally, the prevalence of abdominal obesity was 8.7\% higher than the Australian national average [21]. This study also revealed that $45.9 \%$ of the participants were psychologically distressed using the Kessler 10 score [22] of greater than 15, a rate significantly higher than rural Victorians (31.3\%), all Victorians (34\%) and national (32.7\%) averages [6]. Finally, higher levels of psychological distress were positively correlated with abdominal adiposity.

Abdominal obesity has been suggested to be associated with overstimulation of the hypothalamic-pituitary-adrenal (HPA) axis [23,24] due to chronic stress [25], altering diurnal cortisol secretion. Abnormal regulation of the HPA axis and perceived stress-dependent cortisol levels are strongly related to perturbations of the endocrine axis as well as abdominal obesity with metabolic abnormalities [26].

A complex set of interrelationships occur between lifestyle, anthropometric, psychological and physical activity variables [27]. Of particular interest is the apparent relationship between physical and mental health $[28,29]$. Depressed people generally have lower physical activity levels than people without depression [30,31]. Lower physical activity levels also exacerbate depression resulting in a negative feedback cycle [32]. Conversely, increasing physical activity has the dual benefit of increasing physical fitness and alleviating depression and anxiety [29]. Even without the physical health benefits, increasing physical activity may block negative thoughts, distract people from worries, increase social contact and change the brain chemistry to improve mood [33]. Farming people are seldom thought of as being physically inactive, however the very nature of farming is seasonal, increasingly mechanised, with longer periods of being sedentary and greater reliance on vehicles. Further, opportunities for leisure time activity are reduced since many local sporting clubs have amalgamated or ceased to exist and access to a gymnasium or personal trainers is likely to be difficult due to distance and financial constraints.

The aim of this paper is to describe the pilot testing of the Farming Fit study which will examine the effect of physical activity on psychological health, obesity, impaired glucose tolerance, hypertension and dyslipidaemia in farm men and women.

\section{Methods/Design}

\section{Intervention community}

Inclusion - Farm men and women participating in SFF programs will be recruited. Participants will be recruited from rural Victoria, have been farming for more than 5 years, be aged between 18 and 75 years, speak English and live 10 kilometres or more from a regional centre with a population greater than 10, 000. All study participants will be either overweight or obese as determined by body mass index (BMI) $\geq 25 \mathrm{~kg} / \mathrm{m}^{2}$.

Exclusion - Participants will be excluded from the study if they have a chronic terminal illness or are pregnant or lactating mothers. People with very high levels of psychological distress will be referred for mental health assessment by a health professional (DASS 21 Score depression $>28$ or anxiety $>20$ or stress $>37$ ) [33]. Subjects unable to participate in physical activity will not be eligible to participate.

\section{Preparation for evaluation}

The project design is based on the analysis of data collected by the ongoing SFF program [13]. A logic model of the "farming fit cycle" describes the complex relationship of psychological distress and obesity among farm men and women - see Figure 1 [20]. The study protocol detailed here has been specifically designed to measure how increasing physical activity effects pre-intervention variables (anthropometry, biochemistry, nutritional behavior, physical activity behavior and DASS) [34]. Three data collection time points will be utilised to track participant progress (baseline, 12 weeks and 24 weeks).

\section{Study design}

This is a 6-month quasi-experimental control intervention study. Intervention and control group participants whom fulfil the abovementioned criteria will be selected from SFF programs. SFF program groups (clusters) will

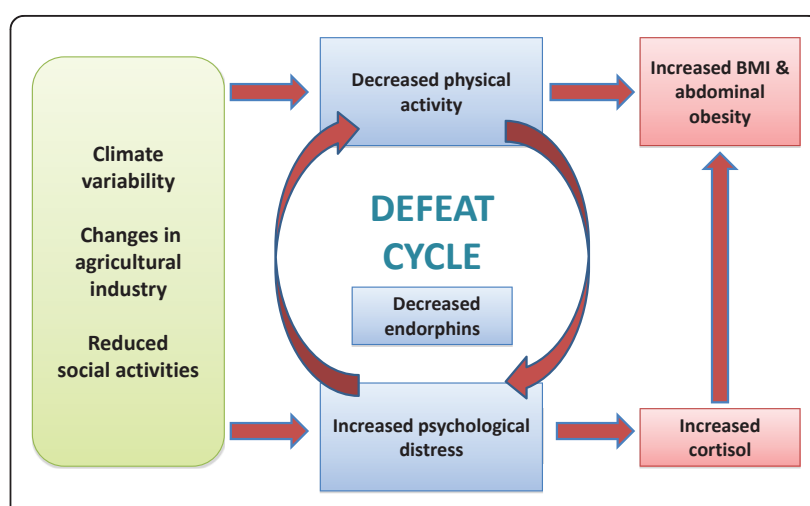

Figure 1 Farming fit study model illustrating the relationship between psychological distress, physical activity and obesity [20]. 
be randomly assigned to control and intervention groups with participants from each cluster being assigned to the same group to minimise the contamination effect.

The proposed intervention includes:

1. An individualised exercise coaching program for the intervention group designed by an exercise physiologist and undertaken over the entire 6-month period.

2. An exercise physiologist/research assistant to provide consultation on individualized exercise programs and coach over the 6-month period.

3. Regular monitoring of exercise activity and exercise goals by phone, email and/or mobile text message.

Participant's anthropometric and basic physical assessments and psychological assessments (DASS 21 scores) will be measured. Venous blood samples following a 10-hour fast will be taken for biochemical analysis of lipids, glucose and cortisol levels. Salivary cortisol measurements will be taken. Diet and physical activity data will be obtained using self reported questionnaires based on previously developed instruments [35,36]. Data collection will be repeated following completion of the 6-month intervention.

Diet and physical activity questionnaires and salivary cortisol samples are to be repeated following an interim 3 -month period of the proposed study as outlined in Table 1. Participants from the intervention group will receive a personalised exercise program and regular phone coaching in order to promote increased physical activity.

Table 1 Data collection and timeline for both intervention and control groups

\begin{tabular}{llll}
\hline Variable & Base line & 3 month & $\mathbf{6}$ month \\
\hline Body weight (kg) & $\checkmark$ & X & $\checkmark$ \\
Height (cm) & $\checkmark$ & X & $\checkmark$ \\
Waist circumference (cm) & $\checkmark$ & X & $\checkmark$ \\
Hip circumference (cm) & $\checkmark$ & X & $\checkmark$ \\
Body fat (\%) & $\checkmark$ & X & $\checkmark$ \\
Blood pressure (mmHg) & $\checkmark$ & X & $\checkmark$ \\
Pulse rate (bpm) & $\checkmark$ & X & $\checkmark$ \\
Fasting blood glucose (mmol/L) & $\checkmark$ & X & $\checkmark$ \\
Fasting blood cholesterol (mmol/L) & $\checkmark$ & X & $\checkmark$ \\
Fasting triglycerides (mmol/L) & $\checkmark$ & X & $\checkmark$ \\
HDL (mmol/L) & $\checkmark$ & X & $\checkmark$ \\
LDL (mmol/L) & $\checkmark$ & X & $\checkmark$ \\
Fasting blood cortisol (nmol/L) & $\checkmark$ & X & $\checkmark$ \\
Salivary cortisol*(nmol/L) & $\checkmark$ & $\checkmark$ & $\checkmark$ \\
DASS score & $\checkmark$ & $\checkmark$ & $\checkmark$ \\
Dietary questionnaire & $\checkmark$ & $\checkmark$ & $\checkmark$ \\
Physical activity questionnaire & $\checkmark$ & $\checkmark$ & $\checkmark$ \\
\hline
\end{tabular}

* Salivary cortisol will be measured by taking 4 readings/day (9 am, 12 pm, $4 \mathrm{pm}$ and $8 \mathrm{pm})$.

\section{Sample size}

To evaluate the intervention, sample size was determined at the power of $80 \%(\beta=0.2)$ with $\alpha$ set at 0.05 for an effect size of a standard deviation change of fasting serum cortisol and/or a 0.5 standard deviation reduction of body weight by the end of the intervention. This required the sample size to be a minimum of 64 participants across the control and intervention groups [37]. By considering the probable retention rate $(\geq 95 \%)$ for a 6 -month intervention the target recruitment number was fixed at a minimum of 35 participants in both the control and intervention groups, requiring recruitment of a total of 70 farming participants meeting the above criteria.

\section{Impact and outcome evaluation}

Impact and outcome evaluation will be achieved by assessing the immediate and longer term effects of the program. Differences in individual and group impacts and outcomes will be measured using longitudinal (3- and 6-month) data with adjustment for baseline data to evaluate any significant changes across the period of the study. The impact outcome of the anthropometric, biochemical and psychological variables of the intervention will be measured using the quasi-experimental study. Differences between intervention and control groups will be statistically ascertained.

\section{Data collection}

Trained research staff will collect the anthropometric, behavioral, dietary and activity data. They will also conduct physical, behavioral and psychological assessments.

\section{Physical Assessment}

A complete health history will be obtained from participants registered in the Farming Fit study. Assessments will include anthropometric measures of height, weight, hip and waist circumference, body fat analysis and biochemical analysis of fasted total serum cholesterol, triglycerides, low-density cholesterol (LDL), high-density cholesterol (HDL), blood glucose and cortisol. Salivary cortisol at different time points across a 24-hour period will also be collected.

\section{Behavioral assessment}

Alcohol habits, activity levels, dietary habits and smoking will be recorded.

\section{Psychosocial assessment}

A validated questionnaire, the 21-question depression, anxiety, stress score (DASS 21) will be used. The DASS 21 is made up of three seven item subscales covering depression, anxiety and stress as specified in Lovibonds and Lovibond [38].

Socio-demographic, health conditions and medication data will be collected from the participant including age, gender, country of origin, health care card using the Victorian Department of Health Service Coordination 
Tool Templates (SCTT) [39]. The type of farming undertaken and residential postcode will also be collected from participants.

\section{Anthropometry}

The simplicity of anthropometry allows it to be used in population-based studies to assess body-composition changes over time, as well as in clinical and field based settings where access to technology is limited. Additionally, if used in combination with biochemistry and body fat \%, anthropometry can identify distinct fat distribution changes [40,41].

Participant weight will be measured (fasted) to the nearest $0.05 \mathrm{~kg}$ using clinically validated $\mathrm{A} \& \mathrm{D}^{\mathrm{TM}}$ precision weight scales (UC-321) taken in light clothing with shoes removed and pockets emptied. Height will be measured using a portable stadiometer $\left(\mathrm{S}+\mathrm{M}^{\mathrm{TM}}\right)$ to the nearest $0.1 \mathrm{~cm}$ with shoes removed and weight distributed evenly on both feet. Waist circumference will be measured to the nearest $0.1 \mathrm{~cm}$ at the umbilicus at the end of a normal expiration and hip circumference taken at the point yielding the maximum circumference over the buttocks using a constant tension Figure Finder Tape Measure ${ }^{\mathrm{TM}}$ [42]. Body Mass Index will be calculated as weight (kilograms) divided by height squared (in meters) to classify obesity and overweight cut-off points [43].

\section{Physical assessments and health condition data}

Physical assessments will be conducted by a trained health professional and will include blood pressure measurement (seated BP measured after at least 5 minutes of rest taken in duplicate one minute apart), pulse rate, body fat percentage (measured by Omron ${ }^{\mathrm{TM}} \mathrm{HBF} 306$ Body Logic Pro Body Fat Analyser). Physical assessments will also include a general medical and behavioural assessment which will collect information on alcohol and tobacco consumption.

\section{Biochemical analysis}

\section{Lipid profile/fasting blood glucose}

Total serum cholesterol, triglycerides, LDL, HDL and fasting blood glucose will be measured to assess metabolic changes associated with abdominal adiposity. Venous blood samples will be obtained following a 10hour overnight fast. Blood samples will be transported in cold storage and analysed at a pathology biochemical laboratory.

\section{Cortisol measurements}

The cortisol response is consistently enhanced under chronic stress conditions and studies have demonstrated that the free cortisol response can serve as a useful index of hypothalamo-pituitary-adrenal axis (HPA) activity [26]. The secretion of cortisol, which is a marker of HPA axis activity, follows a diurnal pattern with peak levels following awakening and declining levels thereafter [44]. In this study, fasting blood cortisol will be measured in addition to salivary cortisol. Assessment of cortisol in saliva is a widely accepted and frequently employed method and is considered a valid and reliable reflection of the respective unbound hormone in blood [45]. Due to several advantages over blood cortisol analyses (e.g. stress-free sampling, less intrusive, lower costs), salivary cortisol assessment is the preferred method in basic research and clinical environments. Salivary cortisol is now an accepted, convenient method that is used to provide an indication of the variation of cortisol secretion throughout the day [45]. Salivary cortisol correlates with serum/plasma cortisol concentrations [45] and is stable at room temperature for up to 7-days [46]. Pregnancy, physical and emotional stress, strenuous activity, infection, injury and illness can increase cortisol levels. A number of drugs can also increase levels, particularly oral contraceptives (birth control pills), hydrocortisone (the synthetic form of cortisol), prednisolone and spironolactone. Therefore, the medication history of study participants will also be collected during the study.

Three groups of saliva samples will be collected using the Salivette sampling device (Sarstedt, Rommelsdorf, Germany) at baseline, three months and six months. Participants will be requested to abstain from smoking, ingesting caffeine, alcohol, food and all fluids, and strenuous physical activity for $1 \mathrm{~h}$ before each saliva collection. A saliva sample will be collected at approximately 09:00 hour, 12:00 hour, 16:00 hour and 20:00 hour. All saliva samples will be stored at room temperature and then spun at 3,000 $\times g$ for 5 min within 5 days of collection. Spun samples will be stored at $-80^{\circ} \mathrm{C}$ until saliva cortisol levels are assayed by Radioimmunology assay (Orion Spectra Cortisol ${ }^{\mathbb{B}}$ ).

\section{Survey methodology \\ Dietary assessment}

Dietary intake will be measured using a one-day food record with household measures collected during a common weekday. The food record will be adapted from survey tools developed by the Dieticians Association of Australia [47]. Portion size of commonly consumed foods will be determined by using the Cancer Council of Victoria Food Frequency Questionnaire [48,49]. Daily energy, macronutrient and micronutrient intake will be determined using Foodworks Professional Edition (version 6.0.2539, Xyris Software, Brisbane, Queensland, Australia). Additionally, an 11 item food frequency questionnaire will be used to determine daily intake of fruit, vegetables, soft drinks/energy drinks, water, sweetened beverages (cordial, fruit juice, fruit drinks, and sports drinks) and weekly intake of snack foods. Frequency of daily food consumption will be categorised as follows: no consumption, 1,2 , 
3,4 and 5 serves or more. Frequency of weekly food consumption will be categorised as follows: $1,2,3,4,5,6,7,8$, 9 and 10 serves or more.

\section{Physical activity behaviours}

Physical activity habits will be measured to assess the influence of this behaviour on body-composition changes. The pre-exercise screening system 2005 of Sports Medicine Australia will be employed to assess the participant's suitability to the particular level of physical activity before personalisation of the program [50]. A questionnaire to collect physical activity data will be prepared by adapting the International Physical Activity Questionnaire (IPAQ) $[35,51]$ and will be administered by a trained researcher at baseline, 3 months and 6 months [35].

Depression, Anxiety and Stress Scale - 21 items (DASS 21)

The Depression, Anxiety and Stress Scale - 21 items (DASS 21) is a set of three self-reported scales designed to measure psychological health status [52]. Each of the three DASS 21 scales contains 7 items, divided into subscales with similar content. The Depression scale consists of items that assess dysphoria, hopelessness, devaluation of life, self-deprecation, lack of interest/involvement, anhedonia, and inertia. The Anxiety scale assesses autonomic arousal, skeletal muscle effects, situational anxiety and subjective experience of anxious affect. The Stress scale is sensitive to levels of chronic non-specific arousal. It assesses difficulty relaxing, nervous arousal, and being easily upset/agitated, irritable/over-reactive and impatient. Scores for Depression, Anxiety and Stress are calculated by summing the scores for the relevant items $[34,38,52]$.

The DASS is a quantitative measure of distress, not a categorical measure of clinical diagnoses. However for clinical purposes it can be helpful to have 'labels' to characterise degree of severity relative to the population. Thus the cut-off scores have been developed for defining mild/moderate/severe/extremely severe scores for each DASS scale (Table 2).

\section{Data entry, handling and statistical analysis}

Data will be tabulated and analysed using PASW ${ }^{\circledR}$ statistics 18 software. Variables will be reported as means with 95\% confidence intervals. Repeated measures ANOVA, paired sample $\mathrm{t}$-tests, multiple linear regression, and binary logistic regression will be used to test for intervention effects after adjusting for relevant co-variables (e.g. sex, age) and baseline scores. Since physical activity scores are likely to reflect a non-normal distribution, comparisons between the sexes at baseline and over time will be performed using the Kruskal-Wallis test. Linear regression analysis will be used to assess the association between body fat percentage, body mass index and cortisol levels. Correlation coefficients between body composition, physical activity, and metabolic variables will be calculated using Pearson's and Spearman's correlation as appropriate.
Table 2 DASS 21 recommended cut-off points [34]

\begin{tabular}{lccc}
\hline & Depression & Anxiety & Stress \\
\hline Normal & $0-9$ & $0-7$ & $\mathbf{0 - 1 4}$ \\
Mild & $10-13$ & $8-9$ & $\mathbf{1 5 - 1 8}$ \\
Moderate & $14-20$ & $10-14$ & $\mathbf{1 9 - 2 5}$ \\
Severe & $21-27$ & $15-19$ & $\mathbf{2 6 - 3 3}$ \\
Extremely Severe & $\mathbf{2 8 +}$ & $\mathbf{2 0 +}$ & $\mathbf{3 7 +}$ \\
\hline
\end{tabular}

Note: the severity labels are used to describe the full range of scores in the population, so 'mild' for example means that the person is above the population mean but probably still below the typical severity of someone seeking help[34].

$P$ values will be considered statistically significant at $\mathrm{P}<0.05$.

\section{Consent and ethics}

All adults participating in the study will be provided with a plain language statement and will provide informed written consent. Ethics approval has been granted for the project by Deakin University Human Research Ethics Committee (2009/215) and the South West Multidisciplinary Ethics Committee (HREC 2009/ 215 dated 03/02/2010). All researchers involved in data collection have had a Victorian Police check undertaken.

\section{Discussion}

Rural populations face poor outcomes in mental health and associated co-morbidities of obesity, diabetes and cardiovascular disease [6-10]. Farming populations are a subset of this group that face additional challenges due to the tyranny of distance, access to health services, stigma associated with mental health issues and the distance-decay effect $[6,12,13]$. They currently have a high mental health burden and higher rates of obesity and associated health consequences [20]. This study aims to determine the effect of physical activity with coaching on psychological health and biochemical levels of serum cholesterol, triglycerides, LDL, HDL, fasting blood glucose and cortisol in a population of overweight and obese farm men and women. The outcome results of this study will be used in further research and service delivery programs for farming communities.

List of abbreviations

SFF: Sustainable Farm Families ${ }^{\mathrm{TM}}$; K-10: Kessler 10; HPA: Hypothalamus Pituitary Adrenal; LDL: Low Density Lipoproteins; HDL: High Density Lipoproteins; DASS-21: Depression, Anxiety, Stress Score-21; WC: waist circumference; BMI: Body Mass Index.

\section{Acknowledgements and Funding}

We would like to acknowledge the generous funding of the beyondblue Victorian Centre of Excellence (bbVCoE) in Depression and Related Disorders (grant reference no. 2009/215). The authors also wish to acknowledge the input of Exercise Physiologist Jason Thomas from Vitality Rehab Pty Ltd and Research Assistant Hannah Simkin from the National Centre for Farmer Health. 


\section{Author details}

'National Centre for Farmer Health, Western District Health Service, Hamilton Vic 3300, Australia. ${ }^{2}$ School of Medicine, Deakin University, Geelong Vic 3217, Australia. ${ }^{3}$ School of Exercise and Nutrition Sciences, Deakin University, Burwood Vic 3125, Australia. ${ }^{4}$ School of Psychology, Deakin University, Geelong Vic 3217, Australia.

\section{Authors' contributions}

SB was an initiator of the original Sustainable Farm Families program, contributed to the design of the study, and specific health assessment protocols. AC, SM, ST, PK and PL participated in the design of the study and drafting of the manuscript. All authors read and approved the final manuscript.

\section{Competing interests}

The authors declare that they have no competing interests.

Received: 15 April 2011 Accepted: 23 May 2011 Published: 23 May 2011

\section{References}

1. Hollingworth S, Burgess $\mathrm{P}$, Whiteford $\mathrm{H}$ : Affective and anxiety disorders: prevalence, treatment and antidepressant medication use. Australian and New Zealand Journal of Psychiatry 2010, 44(6):513-519.

2. Begg S, Vos T, Barker B, Stanley L, Lopez A: Burden of disease and injury in Australia in the new millennium: measuring health loss from diseases, injuries and risk factors. Medical journal of Australia 2008, 188(1):36.

3. Mathers C, Fat DM, Boerma J: The global burden of disease: 2004 update. World Health Organization; 2008.

4. World Health Organisation: Depression. What is depression 2010 [http:// www.who.int/mental_health/management/depression/definition/en/, [cited 2010 20th November].

5. Ustun T, Ayuso-Mateos J, Chatterji S, Mathers C, Murray C: Global burden of depressive disorders in the year 2000. The British Journal of Psychiatry 2004, 184(5):386.

6. Kilkkinen A, Kao-Philpot A, O'Neil A, Philpot B, Reddy P, Bunker S, Dunbar J: Prevalence of psychological distress, anxiety and depression in rural communities in Australia. Australian Journal of Rural Health 2007, 15(2):114-119

7. Wainer J, Chesters J: Rural mental health: Neither romanticism nor despair. Australian Journal of Rural Health 2000, 8(3):141-147.

8. Taylor R, Page A, Morrell S, Harrison J, Carter G: Social and psychiatric influences on urban-rural differentials in Australian suicide. Suicide Life Threat Behav 2005, 35(3):277-90.

9. Bourke L: Toward understanding youth suicide in an Australian rural community. Soc Sci Med 2003, 57(12):2355-65

10. Janus ED, Laatikainen T, Dunbar JA, Kilkkinen A, Bunker SJ, Philpot B, Tideman PA, Tirimacco R, Heistaro S: Overweight, obesity and metabolic syndrome in rural southeastern Australia. Med J Aust 2007, 187(3):147-52

11. Kilkkinen A, Heistaro S, Laatikainen T, Janus E, Chapman A, Absetz P, Dunbar J: Prevention of type 2 diabetes in a primary health care setting. Interim results from the Greater Green Triangle (GGT) Diabetes Prevention Project. Diabetes Res Clin Pract 2007, 76(3):460-2.

12. Miller K, Burns C: Suicides on farms in South Australia, 1997-2001. Aust $J$ Rural Health 2008, 16(6):327-31.

13. Brumby SA, Willder SJ, Martin J: The sustainable farm families project: changing attitudes to health. Rural Remote Health 2009, 9(1):1012.

14. Exworthy M, Peckham S: Access, choice and travel: implications for health policy. Social Policy \& Administration 2006, 40(3):267-287.

15. Zhang J, Conwell Y, Zhou L, Jiang C: Culture, risk factors and suicide in rural China: a psychological autopsy case control study. Acta Psychiatr Scand 2004, 110(6):430-7.

16. Meneese WB, Yutrzenka BA: Correlates of suicidal ideation among rural adolescents. Suicide Life Threat Behav 1990, 20(3):206-12.

17. Singh GK, Siahpush M: Increasing rural-urban gradients in US suicide mortality, 1970-1997. Am J Public Health 2002, 92(7):1161-7.

18. Blackburn J, Brumby S, Willder S, McKnight R: Intervening to improve health indicators among Australian farm families. J Agromedicine 2009, 14(3):345-56.

19. Brumby S, Willder S, Martin J: Milking their health for all its worth? Improving the health of farming families through facilitated learning. Extension Farming Systems Journal 2010, 6(1):1-10.
20. Brumby S, Chandrasekara A, McCoombe S, Kremer P, Lewandowski P: Farming fit? Dispelling the Australian agrarian myth. BMC Research Notes 2011, 4(1):89.

21. Cameron AJ, Welborn TA, Zimmet PZ, Dunstan DW, Owen N, Salmon J, Dalton M, Jolley D, Shaw JE: Overweight and obesity in Australia: the 1999-2000 Australian Diabetes, Obesity and Lifestyle Study (AusDiab). Med J Aust 2003, 178(9):427-32

22. Kessler R, Andrews G, Colpe L, Hiripi E, Mroczek D, Normand S, Walters E, Zaslavsky A: Short screening scales to monitor population prevalences and trends in non-specific psychological distress. Psychological Medicine 2002, 32(06):959-976.

23. Rosmond R, Dallman M, Bjorntorp P: Stress-related cortisol secretion in men: relationships with abdominal obesity and endocrine, metabolic and hemodynamic abnormalities. Journal of Clinical Endocrinology \& Metabolism 1998, 83(6):1853

24. Bjorntorp P: Neuroendocrine factors in obesity. Journal of Endocrinology 1997, 155(2):193

25. Björntorp P: The regulation of adipose tissue distribution in humans. International Journal of Obesity 1996, 20(4):291-302.

26. Wüst S, Federenko I, Hellhammer D, Kirschbaum C: Genetic factors, perceived chronic stress, and the free cortisol response to awakening. Psychoneuroendocrinology 2000, 25(7):707-720.

27. Kyrios M, Moore SM, Hackworth N, Buzwell SA, Crafti N, Critchley C, Hardie E: The influence of depression and anxiety on outcomes after an intervention for prediabetes. Med J Aust 2009, 190(7):S81-5.

28. Teychenne M, Ball K, Salmon J: Associations between physical activity and depressive symptoms in women. Int I Behav Nutr Phys Act 2008, 5:27.

29. Dunn AL, Trivedi MH, Kampert JB, Clark CG, Chambliss HO: Exercise treatment for depression: efficacy and dose response. Am J Prev Med 2005, 28(1):1-8.

30. Brosse AL, Sheets ES, Lett HS, Blumenthal JA: Exercise and the treatment of clinical depression in adults: recent findings and future directions. Sports Med 2002, 32(12):741-60

31. Craft LL, Perna FM: The Benefits of Exercise for the Clinically Depressed. Prim Care Companion J Clin Psychiatry 2004, 6(3):104-111.

32. Rejeski WJ, Shelton B, Miller M, Dunn AL, King AC, Sallis JF: Mediators of Increased Physical Activity and Change in Subjective Well-being: Results from the Activity Counseling Trial (ACT). Journal of Health Psychology 2001, 6(2):159-168

33. Jorm AF, Christensen H, Griffiths KM, Rodgers B: Effectiveness of complementary and self-help treatments for depression. Med J Aust 2002, 176(Suppl):S84-96.

34. Comprehensive Psychological Assessment Centre: DASS 21 - SCORING INSTRUCTIONS. 2010 [http://comprehensivepsychology.com.au].

35. 'Go for your life'. Active living 2010 [http://www.goforyourlife.vic.gov.au/ professionals], [cited 2010 25/10/2010]

36. Sports Medicine Australia: Health conditions and screening 2010 [http://sma org.au/wp-content/uploads/2009/05/new_pre_screening.pdf], [25/10/2010].

37. Norman G, Streiner D: PDQ: Pretty darned quick statistics. Upper Hamilton, Ontario: DC Decker; 2003

38. Lovibond $\mathrm{P}$, Lovibond $\mathrm{S}$ : The structure of negative emotional states: Comparison of the Depression Anxiety Stress Scales (DASS) with the Beck Depression and Anxiety Inventories. Behaviour Research and Therapy 1995, 33(3):335-343.

39. Victorian Government of Health Information: Service Coordination Tool Templates \& Victorian Statewide Referral Form 2010 [http://www.health.vic. gov.au/pcps/coordination/sctt2009.htm]

40. Ross R, Leger L, Morris D, de Guise J, Guardo R: Quantification of adipose tissue by MRI: relationship with anthropometric variables. Journal of applied physiology 1992, 72(2):787.

41. Rankinen T, Kim S, Perusse L, Després J, Bouchard C: The prediction of abdominal visceral fat level from body composition and anthropometry: ROC analysis. International Journal of Obesity 1999 23(8):801-809

42. Utter J, Denny S, Crengle S, Ameratunga S, Robinson E, Clark T, Percival T, Maddison R: Overweight among New Zealand adolescents: Associations with ethnicity and deprivation. International Journal of Pediatric Obesity 2010, 1-6.

43. Balkau B, Charles MA, Drivsholm T, Borch-Johnsen K, Wareham N, Yudkin JS, Morris R, Zavaroni I, van Dam R, Feskins E, Gabriel R, Diet M, Nilsson P, Hedblad B: Frequency of the WHO metabolic syndrome in European 
cohorts, and an alternative definition of an insulin resistance syndrome. Diabetes Metab 2002, 28(5):364-76.

44. Sherwood L: Human physiology: from cells to systems. 10 Davis Drive, Belmont, CA 94002-3098. 7 edition. USA: Brooks/Cole Pub Co, Cengage Learning; 2008.

45. Kirschbaum C, Hellhammer D: Salivary cortisol in psychoneuroendocrine research: recent developments and applications. Psychoneuroendocrinology 1994, 19(4):313-333.

46. Groschl M, Wagner R, Rauh M, Dorr HG: Stability of salivary steroids: the influences of storage, food and dental care. Steroids 2001, 66(10):737-41.

47. Dieticians Association of Australia: Eat better, feel better, move more now 2010 [http://www.healthyweightweek.com.au/files/AHWW\%202011\%20Food \%20and\%20activity\%20record\%20-\%20record\%20page.pdf], [cited 2011 07/ 04/2011].

48. Hodge A, Patterson AJ, Brown WJ, Ireland P, Giles G: The Anti Cancer Council of Victoria FFQ: relative validity of nutrient intakes compared with weighed food records in young to middle aged women in a study of iron supplementation. Australian and New Zealand Journal of Public Health 2000, 24(6):576-583.

49. Woods RK, Stoney RM, Ireland PD, Bailey MJ, Raven JM, Thien FCK, Walters EH, Abramson MJ: A valid food frequency questionnaire for measuring dietary fish intake. Asia Pacific journal of clinical nutrition 2002, 11(1):56-61.

50. K Norton: Sports Medicine Australia (SMA) pre-exercise screening system 2005. Sports Medicine Australia; 2005, A.G.-D.o.H.a. Aging, Editor.

51. Craig CL, Marshall AL, Sjöström M, Bauman AE, Booth ML, Ainsworth BE, Pratt M, Ekelund U, Yngve A, Sallis JF: International physical activity questionnaire: 12 -country reliability and validity. Medicine \& Science in Sports \& Exercise 2003, 35(8):1381.

52. Henry J, Crawford J: The short-form version of the Depression Anxiety Stress Scales (DASS-21): construct validity and normative data in a large non-clinical sample. British Journal of Clinical Psychology 2005, 44(2):227-239.

\section{Pre-publication history}

The pre-publication history for this paper can be accessed here: http://www.biomedcentral.com/1471-2458/11/362/prepub

doi:10.1186/1471-2458-11-362

Cite this article as: Brumby et al:: Reducing psychological distress and obesity in Australian farmers by promoting physical activity. BMC Public Health 2011 11:362.

\section{Submit your next manuscript to BioMed Central and take full advantage of:}

- Convenient online submission

- Thorough peer review

- No space constraints or color figure charges

- Immediate publication on acceptance

- Inclusion in PubMed, CAS, Scopus and Google Scholar

- Research which is freely available for redistribution

Submit your manuscript at www.biomedcentral.com/submit 